\section{Radon activity}

Exposure to large quantities of radioactive radon gas in the home is associated with a high frequency of mutations in a gene in peripheral blood T-lymphocytes. This result, reported by B.A. Bridges et al. in The Lancet (337, $1187-1189 ; 1991)$, is consistent with the controversial idea that leukaemia is more prevalent in countries where the average indoor concentration of radon is high. The blood samples were taken from 20 non-smoking adults from the village of Street, in Somerset, UK, where many homes receive more than the recommended maximum exposure to radon. The authors point out the tentative nature of the finding, which could be accounted for by chance or some confounding factor.

\section{Deep thought}

ANTINEUTRINos radiated from nuclear reactions in the Earth's core could reveal the chemical composition of the deep interior, argue M. Kobayashi and Y. Fukao (Geophys. Res. Lett. 18, $633-636 ; 1991)$. The antineutrinos of interest come from the $\beta$-decay of ${ }^{40} \mathrm{~K}$. The problem is in detecting and identifying them. Twenty seven tons of liquid ${ }^{3} \mathrm{He}$ could help, conclude the author. Collisions of the antineutrinos with the isotope's nuclei produce positrons which radiate scintillation light; with this mammoth detector, one antineutrino may be seen every day. The experiment resembles the neutrino detectors that monitor solar nuclear reactions - hence the article's title, "The Earth as an Antineutrino Star". But enthusiasts should recall that for 20 years the solar results have more perplexed than enlightened.

\section{Spit and polish}

Our saliva contains many proteins, a quarter of them belonging to a family characterized by basicity and an enormous proline content. D. L. Kauffman et al. (Biochemistry $\mathbf{3 0}$, $3351-3356 ; 1991$ ) have now isolated eleven of these from one person (diligent spitting by the senior author) and sequenced six. All or most of them are the products of separate genes, and comparisions with published DNA sequences reveal differences that must be attributed to complex genetic variation. Why we should need so many similar proteins in our saliva is not known, but the conjecture is that the biologically important properties are more composition- than sequencedependent and permit of abundant mutations. The proteins evidently bind to bacteria and may be responsible for attaching them to the teeth and generating plaque. They also, however, lubricate chewing and thus satisfy dentist and epicure alike.
Cosmic censorship is in part the converse, the statement that if the behaviour of spacetime is singular, a black hole must form. But it is stronger: the spacetime outside a black hole must be smooth, its curvature bounded, and the paths of its gravitational waves (or of light rays) must never end, so long as they remain outside any black hole. Although no proof of cosmic censorship has been found, a number of failed attempts to find naked singularities argue in its favour.

Black holes forming in collisions of gravitational waves are initially distorted and dynamic. The waves that are not caught, however, rapidly disperse, and the black hole they leave behind quickly settles down to a final, stationary geometry, characterized solely by its mass and angular momentum. For a given mass, the final black hole can have a maximum angular momentum, roughly that of a ball whose mass and circumference are those of the black hole and which rotates at the speed of light. The assumption of cosmic censorship imposes stringent restrictions on the possible initial spatial geometries, requiring symmetric geometries to have no more angular momentum for their mass than the final black hole. That is, because the waves carry off mass but not angular momentum, the final state would have too much angular momentum to be a black hole (or a set of black holes) and a naked singularity would be the outcome of collapse.

Serious searches have been made for geometries that satisfy the Einstein equations and which violate the conditions that would allow a final black hole state, but they have encountered no success. The equations clearly conspire to avoid geometries that would, at least in this way, remove from singularities the cover of their black holes.

Does adding matter make it possible to evade the cosmic censor? With the simplest kinds of matter, like light, it is no easier to create naked singularities. A collection of particles, however, is difficult to model in a way that correctly accounts for their interactions at high density. If one models matter as, for example, a perfect fluid or as a collection of noninteracting particles, then even in the newtonian theory (in fact, even without gravity) a smooth initial state can have a singular evolution, with points of infinite density. Fluids without pressure (called 'dust') or with unrealistically low pressure cannot keep themselves apart. Several years ago, Kip Thorne suggested an appealing extension of cosmic censorship to exclude such models of matter: singularities that are already present in the newtonian theory will be regarded as uninteresting, the result of the artificial matter, not of the fundamental instability of gravity. Cosmic censorship then requires that if the matter one uses is nonsingular in newtonian gravity, it cannot give rise to naked singularities in the full, relativistic theory. (A similar, but stronger version of the conjecture was proposed last month by Steven Hawking as a bet between him on one side and Thorne and John Preskill on the other. Hawking will regard matter as artificial if it forms singularities without any gravity at all. He bets that cosmic censorship is true for any matter less artificial than that: if matter needs gravity to be singular, its singularities will all be hidden by gravity's event horizons.)

The example that Shapiro and Teukolsky consider is a collection of particles which interact only gravitationally. The particles are initially in the shape of a prolate spheroid a long rounded cylinder - their velocities directed inwards towards its axis. As the particles approach the axis, their density rises and the spacetime curvature - the gravitational field of the particles - increases in tandem. When they reach the axis, their density is infinite, and so is the curvature, but if the spheroid is long enough, no black hole clothes the singularity.

It has long been known that finite cylinders of dust and cylindrical collections of collisionless particles collapse to singularities in the newtonian theory. Thus, by Thorne's criterion, one could say that the naked singularities arising from the collapse of collisionless matter do not violate cosmic censorship.

However, Shapiro and Teukolsky claim that the singularity really extends beyond the collapsing matter, and if it does there is cause for alarm. Outside the matter, the singularity is not simply the result of an infinite density of matter - matter that, by design, is incapable of pushing itself apart. At least it cannot be simply the result of using artificial matter if an observer sees the exterior singularity develop at the same time as the singularity of the matter. For then, even if one changes the matter so that it collides and bounces just before infinite density is reached, it will not be possible to send a signal from the matter to the geometry outside in time to prevent the collapse. A finite speed of light implies that there would be no way to prevent the curvature outside from becoming infinite (see figure).

In fact, if Shapiro and Teukolsky are right, one ought to be able to replace their collisionless particles by a collection of small black holes, and to violate in this way the conjecture that seemed most secure cosmic censorship for vacuum spacetimes. The smaller the black holes, the less often they would collide. One could approximate with arbitrary accuracy the collisionless particles that Shapiro and Teukolsky model, and even spacetimes without matter would have naked singularities.

For now the question remains open. The evidence has more than enough ambiguity to allow prejudice to rule: gravity without matter seems too graceful a theory to allow a breach so crass of a modesty so persistently maintained.

John L. Friedman is in the Department of Physics, University of Wisconsin, Milwaukee, Wisconsin 53201, USA. 